\title{
Algorithmic Approach to Signed Cordiality of Shell Butterfly Networks
}

\author{
V.Jude Annie Cynthia ${ }^{1}$ and E. Padmavathy ${ }^{1, *}$ \\ ${ }^{1}$ Department of Mathematics, Stella Maris College, \\ University of Madras,Chennai, India \\ padmavathyelu@gmail.com
}

September 28, 2021

\begin{abstract}
A shell butterfly graph is defined to be a double shell with exactly two pendant edges at the apex and the right shell with $m$ vertices, the left shell with $l$ vertices. In this paper, we have provided algorithms in order to label the vertices and edges of the graph and have proved that the graph admits signed cordial labeling and signed product cordial labeling.

Key Words : Signed cordial labeling, Signed product cordial labeling, Shell butterfly graphs.
\end{abstract}

\section{Introduction}

The graphs are very powerful structures to clearly show the relationships among the vertices and the edges. One may consider graph theory as a powerful tool for solving various problems in Science, Economics, Medical, and Engineering fields. Now a days graph theory is a key topic for researchers in solving problems in various fields. A graph labeling is an assignment of integers to the vertices

${ }^{*}$ Department of Mathematics, C.T.T.E College for Women,University of Madras, Chennai, India 
or the edges, or both, subject to certain conditions. If the domain is the set of vertices it is vertex labeling. If the domain is the set of edges, then it is edge labeling. If the labels are assigned to the vertices and also to the edges of a graph, such a labeling is called total labeling. Most graph labeling methods trace their origin to one introduced by Rosa in 1967[1]. Labeled graph are becoming an increasingly useful family of mathematical graphs for a broad range of application. According to Beineks and Hegde[10] graph labeling serves as a frontier between number theory and structure of graph. A detail study of variety of applications of graph labeling is given by Bloom and Golomb [5]. A dynamic survey of graph labeling is published and updated every year by Gallian [7]. Cordial labeling is one of the binary labeling which plays an important role in labeling field. An another binary labeling in graph theory towards the development of the research feild is signed cordial labeling and signed product cordial labeling. S-Cordiality with the first letter of Signed Cordiality was introduced by Harray [16]. We can find in the literature that many graphs are proved signed cordial labeling. The concept of signed product cordial labeling was introduced by Babujee et al[18].

A graph $G=(V, E)$ is called signed cordial if it is possible to label the edges with the number from the set $N=\{+1,-1\}$ in such a way that at each vertex $v$, the algebraic product of the labels of the edges incident with $v$ is either +1 or -1 and the inequalities $\left|v_{f}(+1)-v_{f}(-1)\right| \leq 1$ and $\left|e_{f^{*}}(+1)-e_{f^{*}}(-1)\right| \leq 1$ are also satisfied, where $v_{f}(i), i \in\{+1,-1\}$ and $e_{f^{*}}(j), j \in$ $\{+1,-1\}$ are respectively the number of vertices labeled with $i$ and the number of edges labeled with $j$. A graph is called signed-cordial if it admits a signed-cordial labeling.

A vertex labeling of graph $G, f: V(G) \rightarrow\{+1,-1\}$ with induced edge labeling $f^{*}: E(G) \rightarrow\{+1,-1\}$ defined by $f^{*}(u v)=$ $f(u) f(v)$ is signed product cordial labeling if $\left|v_{f}(+1)-v_{f}(-1)\right| \leq$ 1 and $\left|e_{f}^{*}(+1)-e_{f}^{*}(-1)\right| \leq 1$, where $v_{f}(i)$ and $e_{f}^{*}(j)$ are respectively the number of vertices labeled with $i$ and the number of edges labeled with $j$. A graph $G$ is signed product cordial if it admits signed product cordial labeling.

A shell butterfly graph is defined to be a double shell with exactly two pendant edges at the apex and the right shell with $m$ vertices, the left shell with $l$ vertices. 


\section{Literature survey}

The idea of cordial graph was studied by Cahit [6]. Shee and Ho demonstrated that the path union of cycles, Petersen graphs, trees, wheels, unicyclic graphs are cordial [20]. Signed cordial labeling of Petersen graph, complete graph, book graph, jahangir graph and flower graph are studied by Devaraj et al. [8]. Rilwan et al. have proved that the identity graphs admit signed product cordial labeling [12]. Ghosh et al. have investigated on signed product and total signed product cordial labeling of cartesian product between balanced bipartite graph and path [18]. Poulomi et al. have proved that jewel graphs, comb, complete bipartite graphs, gear, web, quadrilateral snake, triangular snake, tadpole, shell are signed product cordial [13]. Sum and union of two fourth power of paths and cycles admits signed product cordial labeling proved by Nada et al.[19]

\section{Main Results}

Shell graphs have interesting and elegant structure to solve problems in graph theory. In this paper, we have provided algorithms to compute vertex labels and edge labels and prove that shell butterfly graphs are signed cordial and signed product cordial. Let $G$ be a shell butterfly graph of order $m$ and $l$ (excluding the apex and two pendent vetrices). The number of vertices of $G$ is $m+l+3$ and the

number of edges of $G$ is $2(m+l)$. Here we fix $m$ to be greater than $l$.

\subsection{Signed Product Cordial Labeling of Shell Butterfly Graphs}

We can label the vertices of the graph $G$ by using the following algorithm. 

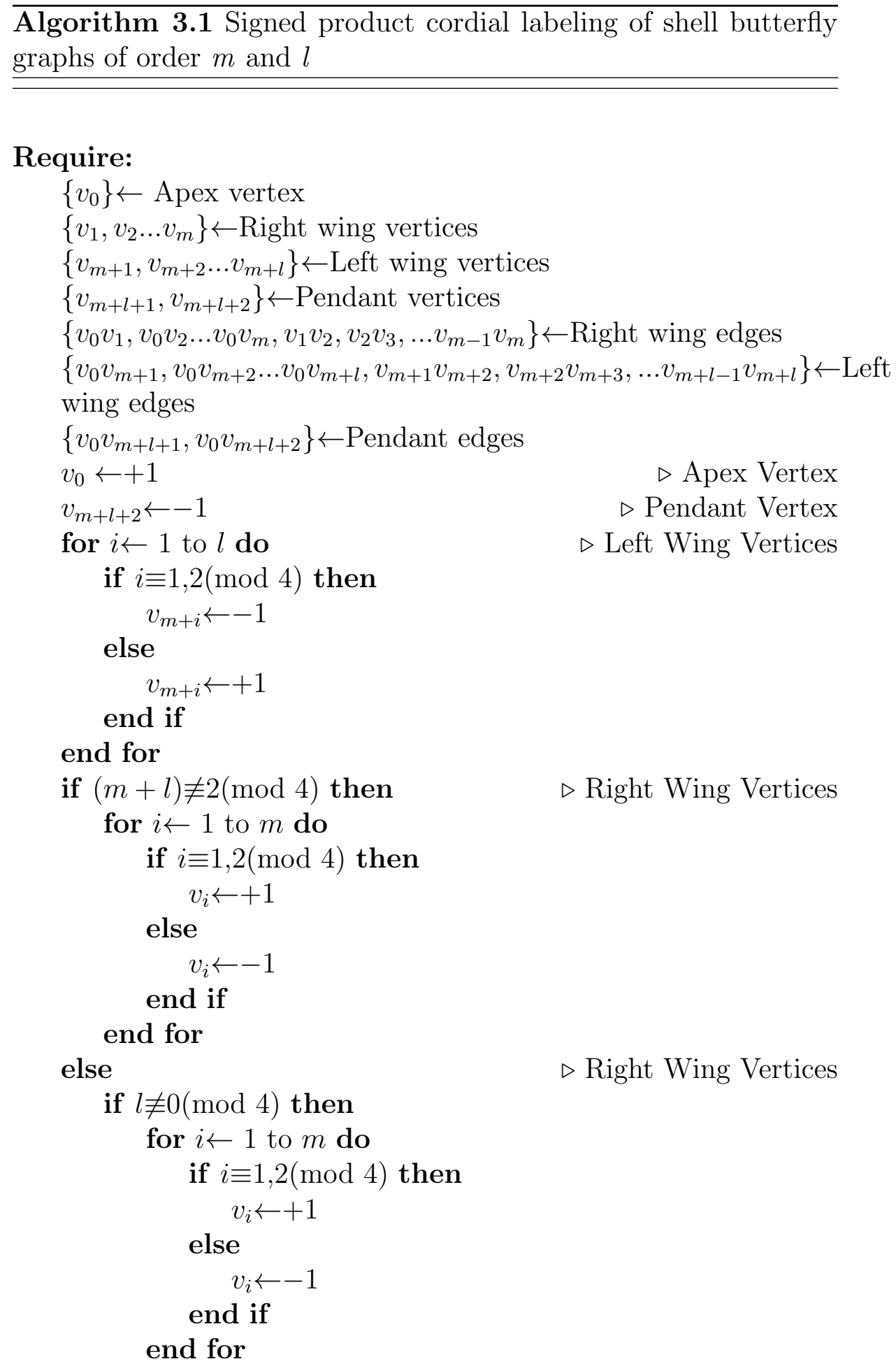
else

for $i \leftarrow 1$ to $m$ do

if $i \equiv 1,2(\bmod 4)$ then

$v_{i} \leftarrow-1$

else

$v_{i} \leftarrow+1$

end if

end for

end if

end if

if $(m+l) \equiv 0(\bmod 4)$ then $\quad \triangleright$ Pendant Vertex

if $l \equiv 0(\bmod 2)$ then

$v_{m+l+1} \leftarrow-1$

else

$v_{m+l+1} \leftarrow+1$

end if

end if

if $(m+l) \equiv 1(\bmod 4)$ then

if $l \equiv 1,2(\bmod 4)$ then

$v_{m+l+1} \leftarrow+1$

else

$v_{m+l+1} \leftarrow-1$

end if

end if

if $(m+l) \equiv 2(\bmod 4)$ then

$v_{m+l+1} \leftarrow+1$

end if

if $(m+l) \equiv 3(\bmod 4)$ then

if $l \equiv 2,3(\bmod 4)$ then

$v_{m+l+1} \leftarrow+1$

else

$v_{m+l+1} \leftarrow-1$

end if

end if

Theorem 1. Shell butterfly graph of order $m$ and $l$ admits signed product cordial labeling.

Proof. Let $G$ be a shell butterfly graph of order $m$ and $l, m \geq l$. Let us define the vertex function of $G$ as $f: V(G) \rightarrow\{+1,-1\}$. 
Following cases arises when we follow the algorithm 3.2.1:

Case 1: $(m+l)$ odd

We get $\left(\frac{m+l+3}{2}\right)$ vertices, labeled with +1 and $\left(\frac{m+l+3}{2}\right)$ vertices, labeled with -1 .

Case 2: $m$ even and $l$ even

In this case we get $\left(\frac{m+l}{2}+2\right)$ vertices, labeled with +1 and $\left(\frac{m+l}{2}+1\right)$ vertices, labeled with -1 .

Case 3: $m$ odd and $l$ odd

We obtain $\left(\frac{m+l}{2}+1\right)$ vertices with the label as +1 and $\left(\frac{m+l}{2}+2\right)$ vertices with the label as -1 .

From the above cases it is clear that the condition $\left|v_{f}(+1)-v_{f}(-1)\right| \leq$ 1 is satisfied.

The induced edge function is $f^{*}: E(G) \rightarrow\{+1,-1\}$. We can observe that the induced edge labels have equal number of +1 and -1 , in all the above three cases. Thus the graph $G$ also satisfies the edge condition $\left|e_{f^{*}}(+1)-e_{f^{*}}(-1)\right| \leq 1$. Hence the graph $G$ admits signed product cordial labeling.

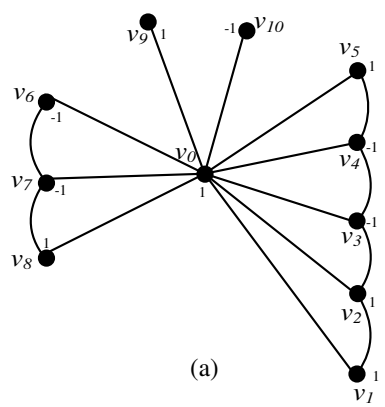

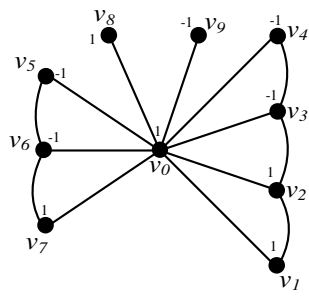

(b)

Figure 1: Signed product cordial labeling of shell butterfly graph

\subsection{Signed Cordial Labeling of Shell Butterfly Graphs}

Edge labels of the graph $G$ using the following algorithm:

$\overline{\text { Algorithm 3.2 Signed cordial labeling of shell butterfly graphs of }}$ order $m$ and $l,(m+l) \not \equiv 3(\bmod 4)$ 


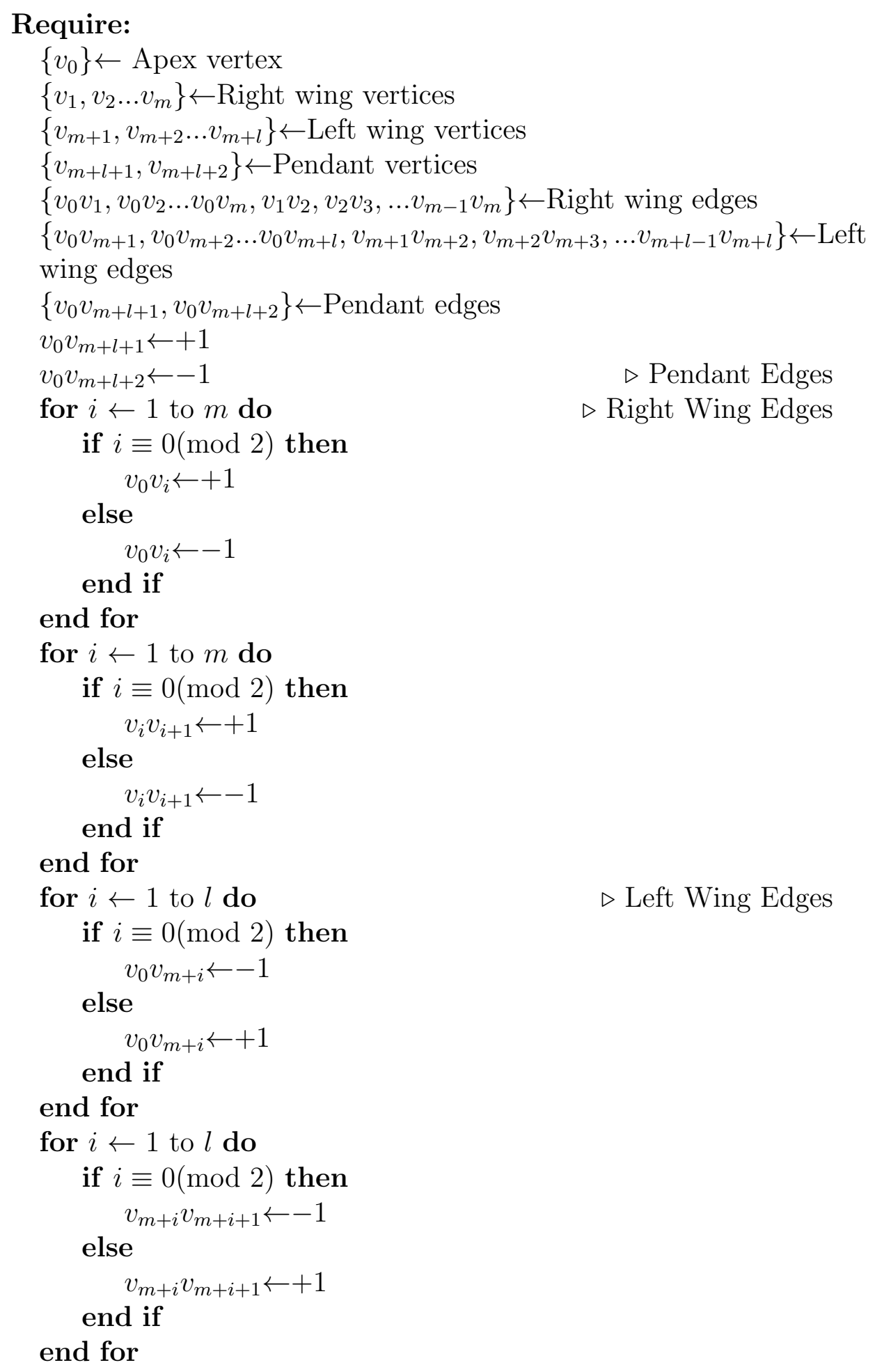


Theorem 2. Shell butterfly graphs of order $m$ and $l,(m+l)$ $\not \equiv 3(\bmod 4)$ admits signed cordial labeling.

Proof. Let $G$ be a shell butterfly graph of order $m$ and $l, m \geq l$. Let us define the edge function of $G, f^{*}: E(G) \rightarrow\{+1,-1\}$ as in the algorithm 3.2.2. Using those procedures, $(m+l)$ edges are labeled with +1 and $(m+l)$ edges with -1 . Thus the graph $G$ satisfies the edge condition $\left|e_{f^{*}}(+1)-e_{f^{*}}(-1)\right| \leq 1$.

The induced vertex function is $f: V(G) \rightarrow\{+1,-1\}$. The induced vertex labels are as follows:

Case 1: $(m+l)$ odd $\left(\frac{m+l+3}{2}\right)$ vertices are labeled with +1 and $\left(\frac{m+l+3}{2}\right)$ vertices are labeled with -1 , in this case see Figure 3.2(a).

Case 2: $(m+l) \equiv 2(\bmod 4)$

In this case, $\left(\frac{m+l}{2}+2\right)$ vertices are labeled with +1 and $\left(\frac{m+l}{2}+1\right)$ vertices are labeled with -1 .

Case 3: $(m+l) \equiv 0(\bmod 4)$

$\left(\frac{m+l}{2}+1\right)$ vertices are labeled with +1 and $\left(\frac{m+l}{2}+2\right)$ vertices are labeled with -1 . See Figure 3.2(b).

From the above cases we can see that the inequality $\left|v_{f}(+1)-v_{f}(-1)\right| \leq$ 1 is satisfied. Hence the graph $G$ admits signed cordial labeling.

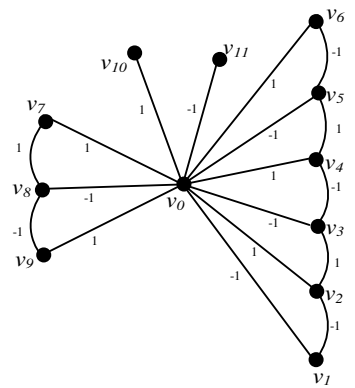

(a)

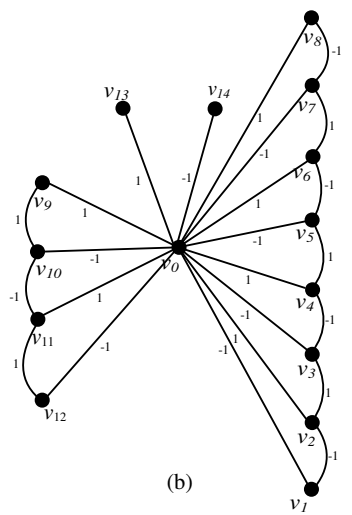

Figure 2: Signed cordial labeling of shell butterfly graphs 


\section{Conclusion}

In this paper, we have provided algorithms to prove that the shell butterfly graph admit both signed cordial labeling and signed product cordial labeling. Further the signed cordial and signed product cordial labeling of interconnection networks are under study.

\section{References}

[1] A. Rosa, On certain valuations of the vertices of a graph, Theory of graphs (Internat.Symposium, Rome, July 1966), Gordon and Breach, N.Y. and Dunod Paris, 349-355, 1967.

[2] A. H. Rokadi, Product cordial labeling of double wheel and double fan related graphs, Kragujevac Journal of Mathematics Volume, 43(1), 7-13, 2019.

[3] D. Antony Xavier and C.J. Deeni, Bloom graph, International Journal of Computing Algorithm, 521-523, 2017.

[4] G.H. Yang, Q.D. Kang, Z.H. Liang and Y.Z. Gao, On the labeling of some graphs, J. Combin. Math. Combin. Comput., 22, 193-210, 1996.

[5] G.S. Bloom and S.W Golomb, Applications of numbered undirected graphs, Proceedings of IEEE, 562-570, 1977.

[6] I. Cahit, Cordial Graphs: A weaker version of graceful and harmonic graphs, Ars Combinatoria, 201-207, 1987.

[7] J.A. Gallian, A dynamic survey of graph labeling, The Electronics Journal of Combinatorics, 17, DS6, 2019.

[8] J. Devaraj and P. Prem Delphy, On signed cordial graph, International Journal of Mathematical sciences and application, 1, 2011.

[9] Jayapal Baskar Babujee, Shobana Loganathan, On signed product cordial labeling, Scientific Research Journal on Applied Mathematics, 2, 1525-1530, 2011. 
[10] L.W. Beineke and S.M. Hegde, Strongly Multiplicative graphs , Discuss. Math. Graph Theory, 21, (2001), 63-75.

[11] M. Santhi and James Albert, Signed product cordial in cycle related graphs, International Journal of Mathematics and Computer Application Research, 5, 29-36, 2016.

[12] N. Mohamed Rilwan and A. Syed Hussain, Prime cordial and signed product cordial labeling on identity graph, Advances in Mathematics: Scientific Journal, 8, 749-753, 2019.

[13] Poulomi Ghosh and Anitha Pal, Special classes of signed product cordial graphs, International Journal of Computer Science and Information Security, 15, 2017.

[14] P. Lawrence Rozario Raj and R. Lawrence Joseph Manoharan, Face and total face signed product cordial labeling of planar graphs, Asia Pacific Journal of Research, 1, 2016.

[15] P. Lawrence Rozario Raj and R. Lawrence Joseph Manoharan, Signed product cordial graphs in the context of arbitrary supersubdivision, Scientia Magna, 8, 77-87, 2012.

[16] P.P. Ulaganathan, B. Selvam and P. Vijaya kumar, Signed product cordial labeling in duplicate graphs of bistar, double star and triangular ladder Graph, International Journal of Mathematics Trends and Technology, 33, 2016.

[17] S.M.Hedge and S.Shetty, On magic graphs, Australasian J. Combinatorics, 27, 277-284, 2003.

[18] Sumonta Ghosh and Anita Pal, Signed product and total signed product cordial labeling of cartesian product between balanced bipartite graph and path, Advanced Computational and Communication Paradigms, 706, 515-522, 2018.

[19] Shokry Nada, Amani Elrayes, Ashraf Elrokh and Aya Rabie Signed product cordial of the sum and union of two fourth power of paths and cycles, Machine Learning Research, 4, 4550, 2019.

[20] S C Shee , Y S Ho , The cordiality of path-union of n copies of a graph, Discrete Math, 221-229, 1996. 